\title{
A Simulation Based Performance Comparison of Routing Protocols (Reactive and Proactive) For Random Waypoint Mobility Model
}

\author{
Sukhpreet kaur (M.Tech Student),Chandan Sharma (Ph.d. Candidate), \\ Sri Guru Granth Sahib World University, Fatehgarh Sahib \\ Guru Nanak Dev University, Amritsar, india(pb.)
}

\begin{abstract}
Mobile Ad hoc Network is collection wireless mobile nodes which dynamically form a temporary network] =without the use of existing network infrastructure centralized administration. Due to dynamic property of mobiles nodes in MANET, They require good routing protocols. This paper analyzes the effect of random based mobility models on the performance of Proactive Routing Protocol (DSDV-Destination Sequence Distance Vector) and Reactive Routing Protocol (AODV- on Demand Distance Vector Routing Protocol, and (OLSR-Optimized Link State Routing Protocol). Performance Analysis is done with respect to receive rate and packet received for varying node densities.
\end{abstract}

\section{Introduction}

Mobile ad-hoc networks (MANET) are collections of wireless mobile nodes dynamically forming a temporary network without the use of any pre-defined network infrastructure or centralized administration. [6].MANETs as shown in fig (1) have characteristics that network topology changes very rapidly and unpredictably in which many mobile nodes moves to and from a wireless network without any fixed access point where routers and hosts move, so topology is dynamic[7].

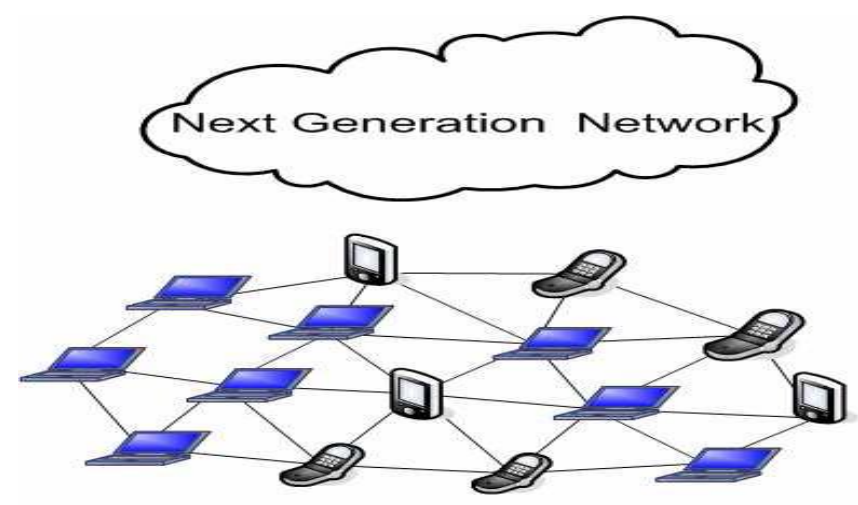

Fig. 1 Mobile Ad hoc Network- MANET[7]

A central challenge in the design of ad hoc networks is the development of dynamic routing protocols that can efficiently find routes between two communicating nodes. Several protocols exist and those can be divided into three main categories, Reactive, Proactive and Hybrid Protocols. Reactive protocols are characterized by nodes acquiring and maintaining routes on-demand. Proactive protocols are categorized by all nodes maintaining routes to all destinations in the network at all times. They are also called table driven protocols[5].The topology of the ad hoc network depends on the transmission power of the mobile nodes which may change with time . Routing Protocols for ad hoc network need to perform a set of route identification and route reconfiguration [8].

Now days mobile ad hoc network have robust and efficient operation in mobile wireless networks as it can include routing functionality into mobile nodes which is more than just mobile hosts and reduces the routing overhead and saves energy[3].

For other nodes . MANET are very useful when infrastructure is not available, impractical or expensive because it can be rapidly deployable without prior planning or any existing infrastructure.

This paper is structured as follows: Section II discusses related work. In Section III we briefly review MANETs routing protocols and mobility models. Section IV provides details of simulation parameters and performance metrics, Section V gives Simulation Set up. Finally, in Section VI Results are discussed followed by the conclusions. 


\section{Related Work}

Performance comparison among some set of MANET routing protocols (proactive, Reactive, Hybrid) is already done by the researchers such as among PAODV , AODV DSR and CBRP [2], among DSR, AODV, and DSDV,[3], among OLSR, DSDV, and FSR[4], in [5] among AODV, DSDV and ZRP , among AODV , DYMO, ZRP, OLSR[6] , among AODV, OLSR, and TORA [7].This paper presents the performance comparison of OLSR, AODV, and DSDV routing protocols where OLSR ,AODV ,DSDV are prominent protocols of proactive and reactive respectively. In this paper, we simulate routing protocols and give a brief discussion on their respective behaviour according to different environment.

\section{Manet Routing Protocols}

Routing protocols for wireless ad hoc networks can be classified into the three categories: On-demand ( or Rective) protocols, Table - driven ( or Proactive) protocols, Hybrid protocols. Figure 2 shows the basic classification of the routing protocols in MANETs.

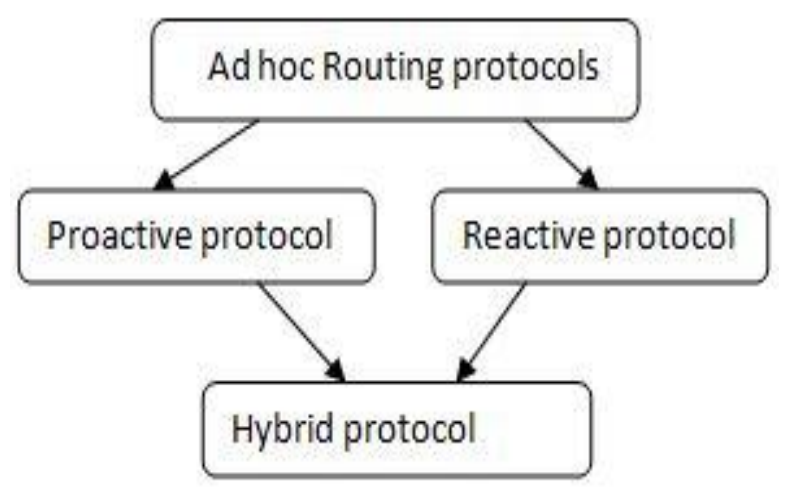

Fig.2 Basic classification of the routing protocols in MANETs.

On-demand (reactive) protocols: In contrast to table driven routing protocols, on demand compute the route to a specific destination only when needed, so a routing table containing all the nodes as entries does not have to be maintained in each node. When a source wants to send packet to a destination, it invokes a route discovery mechanism to find the path to the destination. The route remains valid till the destination is reachable or until the route is no longer needed. Reactive protocols can be classified into two categories: source routing and hopby-hop routing[9].Examples are Reactive protocols AODV, DSR [5].

Table driven (proactive) protocol: Proactive protocols maintain the routing information consistently up-todate from each node to every other nodes in the network. The main function of proactive routing protocols maintain its table in order to store routing information[5,7]. These tables are periodically updated and/or if the network topology changes[9].

Hybrid Protocols:- Hybrid routing protocols are a new generation of protocol, which are both proactive and reactive in nature. These protocols are designed to increase scalability by allowing nodes with close proximity to work together to form some sort of a backbone to reduce the route discovery overheads. This is mostly achieved by proactively maintaining routes to near by nodes and determining routes to faraway nodes using a route discovery strategy. Most hybrid protocols proposed to date are zone-based, which means that the network is partitioned or seen as a number of zones by each node. Others group nodes into trees or clusters[9].

1) AODV (Ad hoc On-Demand Routing Protocols):- it is reactive protocol that improve the DSDV in sense of minimizing the number of required broadcasts by creating routes on demand basis as opposed to maintaining a complete list of routes[8]. AODV use a broadcast route discovery mechanism where source node initiate a route dicovery method by broadcasting a route request (RREQ) packet to its neighbours 


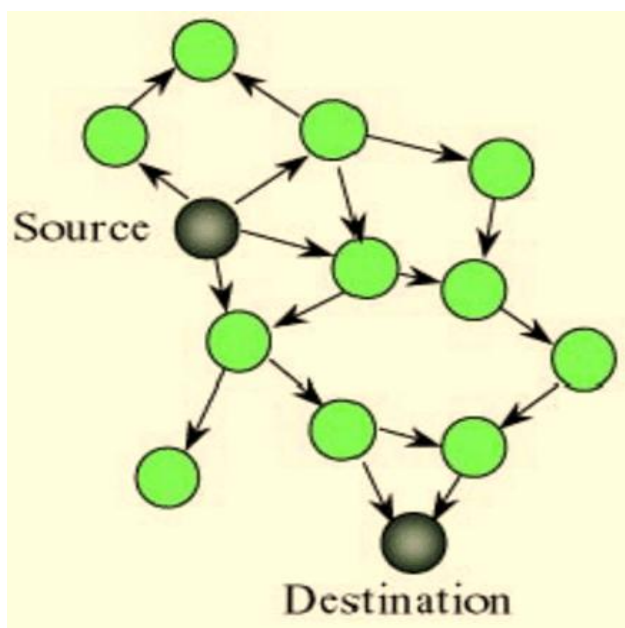

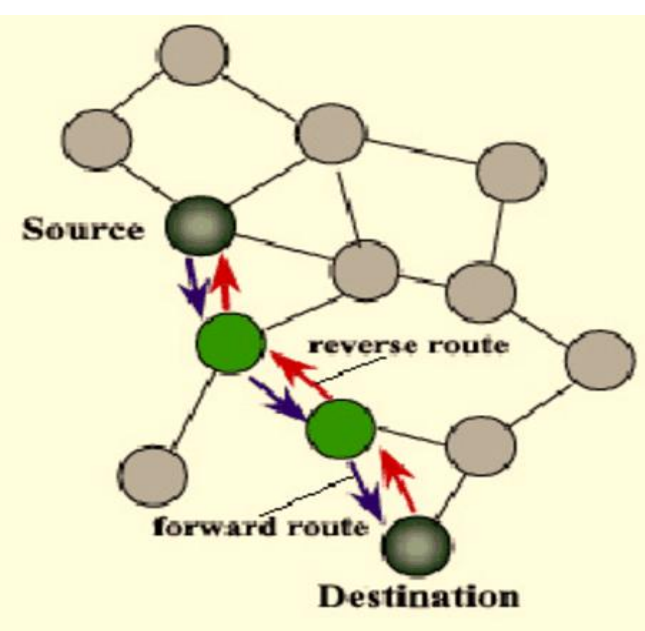

Fig. 3 Route Discovery - (i) RREQ (ii) RREP [5].

The RREQ packet contain a sequence number and a broacast message id. Each neighbor satisfied with the RREQ replies with the route reply (RREP) packet adding one in the hop count field. Unlike DSDV, in AODV if a node cannot satisfy the RREQ, it keeps track of the necessary information in order to implement the reverse and forward path setup that will accompany the transmission of the RREP. The source sequence number is used to maintain freshness information about the reverse route to the source and the destination sequence number specifies how fresh a route to the destination must be before it can be accepted by the source. The source node can begin data transmission as soon as the first RREP is received. Hence, the first sending of data packet to the destination is delayed due to route discovery process[3].

2) DSDV (Destination Sequenced Distance Vector Routing):- This protocol is based on classical BellmanFord routing algorithm designed for MANETS. Each node maintains a list of all destinations and number of hops to each destination. Each entry is marked with a sequence number. It uses full dump or incremental update to reduce network traffic generated by rout updates. Each mobile node maintains a routing table in which all the possible destinations and the number of hops to them in the network are stored. The entries in the table may change extremely dynamically so the advertisements might be made quite often[8]. It is quite suitable for creating ad hoc network with small number of nodes. Since no formal specification of this algorithm is present , there is no commercial implementation of this algorithm. DSDV requires a regular update of its routing tables, which uses up battery power and a small amount of bandwidth even when the network is idle. Whenever the topology of the network changes, a new sequence number is necessary before the network re-converges. Thus, DSDV is not suitable for highly dynamic networks. (As in all distance-vector protocols, this does not perturb traffic in regions of the network that are not concerned by the topology change)[5].

3) OLSR (Optimized Link State Routing):- is based on link state algorithm and it is proactive in nature. OLSR is an optimization over a pure link state protocol as it squeezes the size of information send in the messages, and reduces the number of retransmissions. It provides optimal routes in terms of number of hops. For this purpose, the protocol uses multipoint relaying technique to efficiently flood its control messages .Unlike DSDV and AODV, OLSR reduces the size of control packet by declaring only a subset of links with its neighbors who are its multipoint relay selectors and only the multipoint relays of a node retransmit its broadcast messages. Hence, the protocol does not generate extra control traffic in response to link failures and node join/leave events. OLSR is particularly suitable for large and dense networks. All mobile nodes maintain the routing table that contains routes to all reachable destination nodes. OLSR does not notify the source immediately after detecting a broken link and source node comes to know that route is broken when the intermediate node broadcasts its next packet[6].

Mobility Models:- The mobility model is designed to describe the movement pattern of mobile user, and how their location, direction of movement, pause distribution, speed and acceleration change over time. Mobility model is a key parameters that researchers have to consider who they want to analyze the performance of the certain protocols in their simulation environment. The selection of different mobility model can have a major impact on the selection of a routing scheme and can thus influence performance. 


\section{Random Waypoint Mobility Model:-}

The randon waypoint mobility model introduce a specific pause time between movement i.e. change in direction and speed. The random waypoint model is the most popular mobility model employed in contemporary research and can be considered a foundation for building other mobility models.

\section{Random Walk Mobility Model:-}

The random walk mobility model is the simplest mobility model, generating completely random movement patterns. It was designed for simulation in which the movement patterns of mobile nodes are completely unpredictable. In this mobility model, an MN moves from its current location to a new location by randomly choosing a direction and speed in which to travel in this model a mobile node is initially placed in a random location in the simulation area and then moved randomly chosen direction between [0.2pi] at a random speed between [SpeedMin, SpeedMax].

\section{Random Direction Mobility Model:-}

In Random Direction Mobility Model MNs using the Random Waypoint Mobility Model often choose new destinations, and the probability of choosing a new destination that is located in the center of the simulation area, or requires travel through the middle of the simulation area, is high. The MNs moving with the Random Waypoint Mobility Model appear to converge, disperse, converge again, etc. In order to alleviate this type of behavior and promote a semi-constant number of neighbors, the Random Direction Mobility Model was developed[8].

\section{Simulation Parameters:-}

\begin{tabular}{|c|c|}
\hline statistic & Value \\
\hline Simulator & NS3 \\
Protocols studies & AODV, DSDV,OLSR \\
Scenario size & $1000 * 1000$ \\
Number of nodes & 30 to 50 \\
Node movement model & Random waypoint \\
WIFI PHY-Standard & $\mathbf{8 0 2 . 1 1 b}$ \\
Node speed(m/s) & 20 \\
Transmission power & 7.5 to $9.5 \mathrm{dbm}$ \\
Simulation time (sec) & 100 to 200 \\
Pause time(m/s) & 0 to 0.5 \\
& \\
& \\
\hline
\end{tabular}

Table.1 Simulation Parameters

\section{Simulation Environment:-}

Simulation environment consist of 30 wireless mobile nodes which are placed uniformly and forming a mobile ad hoc network, moving about over a 1000 x 1000 . All mobile nodes configured to run ad hoc on demand distance vector protocols or destination source distance vector protocols or Optimized link state routing protocol. The simulation were performed using Network Simulators 3 in Linux Ubuntu. The 'Random Waypoint Model' in a rectangle field of $1000 \times 1000$ with 20 nodes to 50 nodes with $20 \mathrm{~m} / \mathrm{sec}$ as a maximum speed and pause times $5 \mathrm{~m} / \mathrm{sec}$. simulation time for the whole scenario was 100 to $200 \mathrm{sec}$

\section{Result and Discussion}

In this section we compare the three protocols routing protocols. To evaluate the performance of AODV, DSDV, and OLSR

routing protocols in same simulation environment with ( 30 to 50 nodes). Simulation results are collected from different scenarios of three protocols. The simulation results are shown in the following section in the form of graph. In the first three figure we compared AODV, OLSR, and DSDV with variable node density 30, simulation time was $100 \mathrm{sec}$ and pause time $0.5 \mathrm{~m} / \mathrm{s}$. In next figures compared with 50 nodes, simulation time was $200 \mathrm{sec}$ and pause time is zero (0). 


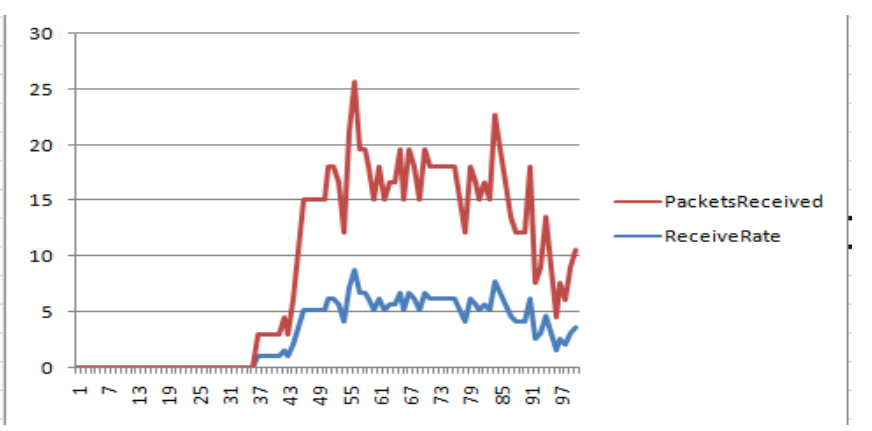

Fig. 3 ReceiveRate and packetRecieved when using AODV with 30 nodes

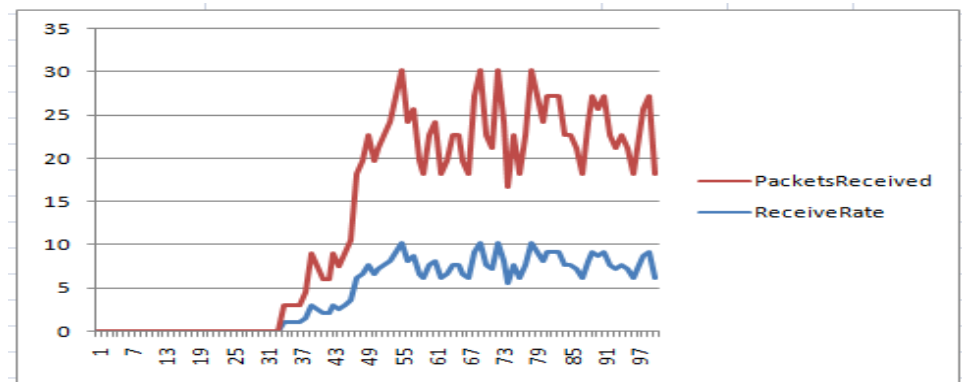

Fig.4ReceiveRate and packetRecieved when using OLSR with 30 nodes

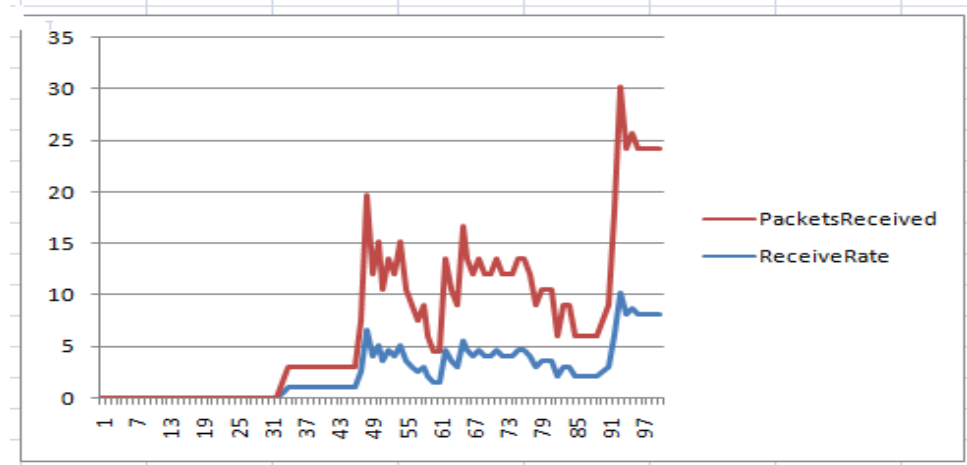

Fig.5ReceiveRate and packetRecieved when using DSDV with 30 nodes

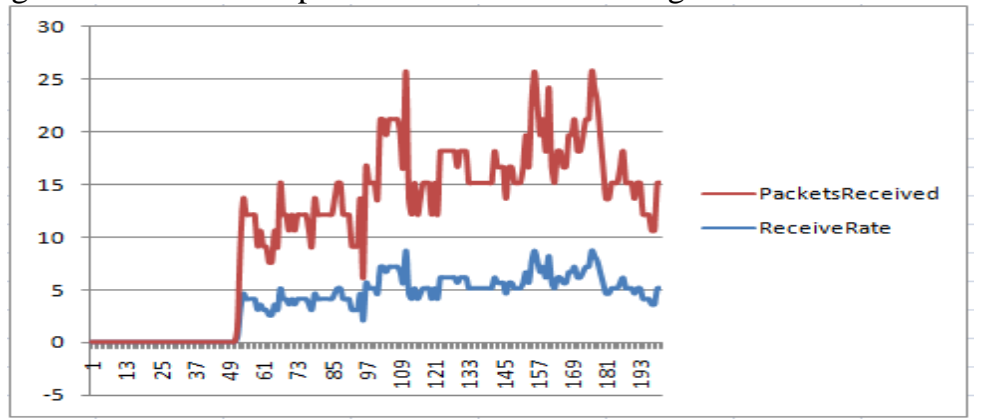

Fig.6ReceiveRate and packetRecieved when using AODV with 50 nodes

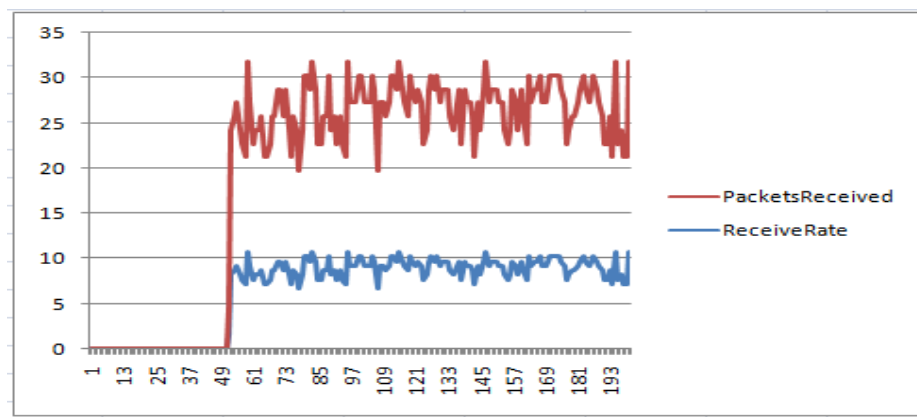

Fig.7ReceiveRate and packetRecieved when using OLSR with 50 nodes 


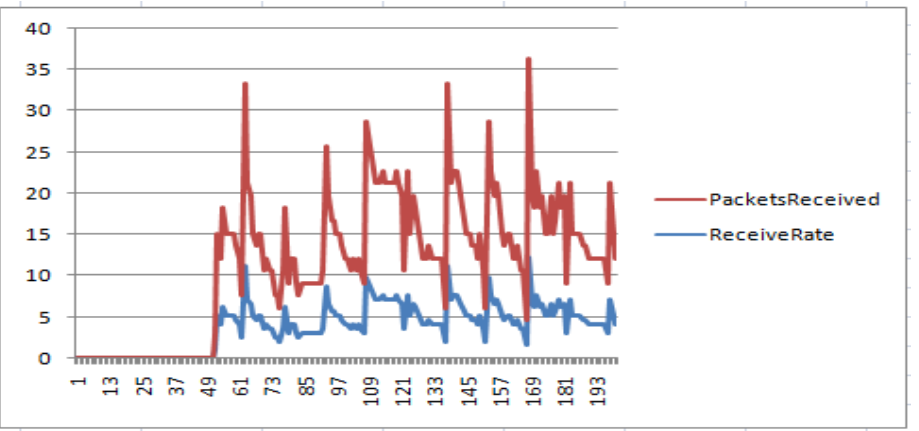

Fig .8ReceiveRate and packetRecieved when using DSDV with 50 nodes

\section{Conclusion:-}

In this paper, performance evaluation of random based mobility model on three routing protocols AODV, OLSR, DSDV is compared with respect to two performance metrices using the differents parameters. OLSR performed better for both metrices than AODV and DSDV when the simulation time is $100 \mathrm{sec}$ with 30 nodes. DSDV performed better for 50 nodes than OLSR And AODV. From simulation result, its observed that AODV has poor performance in both case.

\section{References:-}

[1]. Robinpreet Kaur \& Mritunjay Kumar Rai “A Novel Review on Routing Protocols in MANETs”, Undergraduate Academic Research Journal (UARJ), ISSN : 2278 - 1129, Volume-1, Issue-1, 2012

[2]. Boukerche, "Performance Evaluation of Routing Protocols for Ad Hoc Wireless Networks," Mobile Networks and Applications, pp. 9, 333-342, Kluwer Academic Publishers, 2004.

[3]. Shrikant Upadhyay “ comparison and performance analysis of reactive type DSR, AODV and proactive type mobile ad-hoc network, using NS-2 simulator"Journal of Engineering and Computer Innovations Vol.2 (10), pp 36-47, March 2012.

[4]. D. Mahmood,N. javaid , U. Qasim , Z. A. Khan "Routing Load of Route Calculation and Route Maintenance in Wireless Proactive Routing Protocols", seventh International Conference on Broadband, wireless Computing, communication and applications,2012.

[5]. Savita Gandhi SMIEEE1, Nirbhay Chaubey MIEEE2, Naren Tada3, Srushti Trivedi3, "Scenario-based Performance Comparison of Reactive, Proactive \& Hybrid Protocols in MANET", International Conference on Computer Communication and Informatics , 2012.

[6]. Md. Arafatur Rahman and Farhat Anwar, Jannatul Naeem and Md. Sharif Minhazul Abedin "A Simulation Based Performance Comparison of Routing Protocol on Mobile Ad-hoc Network (Proactive, Reactive and Hybrid)", International Conference on Computer and Communication Engineering,2012.

[7]. Nadin Qasim, Fatin Said, Hamid Aghvami, "Mobile Ad Hoc Networking Protocols Evaluation through Simulation for Quality of Service", IAENG International Journal of Computer Science,2009.

[8]. Rashmi Rohankar, Rinkoo Bhatia, Vineet Shrivastava, Deepak Kumar Sharma, "Performance Analysis of Various Routing Protocols (Proactive and Reactive) for Random Mobility Models of Adhoc Network", 1st Int'l Conf. on Recent Advances in Information Technology, 2012.

[9]. Mehran Abolhasan, Tadeusz Wysocki, Eryk Dutkiewicz "A review of routing protocols for mobile ad hoc networks" 